\title{
Agora: a semantic overlay network
}

\section{Geert Deconinck* and Koen Vanthournout}

\author{
K.U. Leuven - Electrical Engineering Department (ESAT) \\ Kasteelpark Arenberg 10 \\ B-3001 Leuven, Belgium \\ Fax: +32 16321985 \\ E-mail: Geert.Deconinck@esat.kuleuven.be \\ E-mail: Koen.Vanthournout@gmail.com \\ *Corresponding author
}

\begin{abstract}
Overlay networks are increasingly receiving attention as a scalable and distributed platform to realise resource discovery for file-sharing applications. However, their potential application range is much broader, as they can even serve as a communications infrastructure for distributed control and automation, e.g., underlying the electricity infrastructure. This requires the support of generic primitives such as, e.g., attribute-based semantic routing. Due to their focus on file sharing, today's systems do not or inefficiently support attribute-based semantic routing. In response, this paper proposes an innovative overlay class that supports attribute-based, semantic addressing. Agora, a set of algorithms, provides an implementation of such semantic overlay network. The simulation results confirm the claims.
\end{abstract}

Keywords: overlay networks; self-organisation; semantic routing; critical infrastructures.

Reference to this paper should be made as follows: Deconinck, G. and Vanthournout, K. (2009) 'Agora: a semantic overlay network', Int. J. Critical Infrastructures, Vol. 5, Nos. 1/2, pp.175-195.

Biographical notes: Geert Deconinck is a Full Professor (hoogleraar) at K.U. Leuven (Belgium). As a staff member of Electrical Energy and Computing Architectures (ESAT/ELECTA), he performs research on designing dependable system architectures for industrial automation and control, assessing their dependability attributes and characterising infrastructure interdependencies. $\mathrm{He}$ received his MSc in Electrical Engineering (1991) and his $\mathrm{PhD}$ in Engineering (1996) from K.U. Leuven and was a Postdoctoral Fellow of the Fund for Scientific Research - Flanders (1997-2003). He is a member of the Royal Flemish Engineering Society and a senior member of the IEEE (Reliability, Computer and Power Engineering Societies).

Koen Vanthournout received his Master's degree in Electrical Engineering (1999) from the Groep T Hogeschool of Leuven, Belgium, and his Master's degree in Artificial Intelligence (2000) from K.U. Leuven, Belgium. He obtained his PhD in Electrical Engineering from K.U. Leuven in 2006. His research interests include automation, self-organising systems and networked distributed systems. He is currently a Senior Embedded Software Engineer with KLA-Tencor. 


\section{Introduction}

Overlay networks are directed graphs built on top of an all-to-all communication network, in which the edges define which node is aware of other nodes. They constitute a communication framework on which distributed applications can be realised, the most popular of which is file-sharing resource discovery (commonly referred to as Peer-to-Peer or P2P networks). Two main classes of overlays can be distinguished:

1 Unstructured overlays - The first generation of P2P systems, such as Gnutella or Freenet (Clarke et al., 2001), impose structure on their overlay graph and are therefore named unstructured overlays. These unstructured overlays are often used to realise file-sharing resource discovery. They implement a clear text-based search usually based on flooding, random forwarding or other blind-search strategies; such strategies typically have a low efficiency.

2 Distributed Hash Table (DHT) overlays - In order to improve the search efficiency of unstructured overlays, a second generation emerged: DHT overlays, e.g., Content Addressable Network (CAN) (Ratnasamy et al., 2001), Chord (Dabek et al., 2001), Pastry (Rowstron and Druschel, 2001), PGrid (Aberer et al., 2004), etc. DHT P2P systems assign an identifier (ID) to both nodes and resources, based on the nodes' network addresses and the (static) resources' (clear-text) descriptions, respectively. The nodes are then positioned in a structured graph which reflects the ID space and allows deterministic routing. Resources or references to resources are located at the node with the closest ID. If the ID of a resource is known, then the search for that resource equals the search to the node with the closest ID, which is deterministic and highly efficient. The disadvantage is the use of fixed resource IDs, which prohibits attribute-based search or the reflection of the dynamic properties of resources (e.g., the load of a server) in the naming system, a restriction which stems from the focus on file sharing. The semantics of the resource names are not reflected in the naming system.

Although today's overlay network research focuses on resource discovery for file sharing, the application of overlay networks could be expanded as a communications infrastructure for genuinely distributed systems in general, including distributed automation and control (an example of which can be found in Vanthournout et al., 2005a). This requires overlay networks to provide a semantic routing primitive (Vanthournout et al., 2005b), i.e., the ability to route a message to a node matching a certain description, rather than a network address. Semantic routing can then be used to realise resource discovery, event notification, data dissemination, etc.

In order to serve this broad range of applications, the descriptions attached to a semantic routing message must be as generic as possible and support easy machine-based handling, i.e., these descriptions must be attribute-based and be able to describe devices with dynamic properties. This is not the case for today's systems. The unstructured overlays use text-based descriptions, which are not easily processed by machines, while using non-efficient overlay search strategies. DHT overlays are highly efficient, but have the drawback of using numerical IDs, which are unable to reflect semantics or dynamic properties. 
In response, we propose the concept of semantic overlay networks as a new class of overlay networks compliant to the requirements described above (Section 2) together with a design for such a semantic overlay, named Agora (Section 3). Finally, the simulation results, confirming Agora's semantic overlay properties, are presented in Section 4.

\section{Semantic overlay networks}

In order to improve the efficiency of unstructured networks, DHT overlays impose structure on their graph by means of self-organisation, which must support efficient (nonblind) searches. On the other hand, overlays benefit from having a small diameter, which ensures short paths for one node to reach another. This combination of the best of both random graphs (low diameter, but low clustering) and regular graphs (high clustering, but also high diameter) is named small-world graphs (high clustering, low diameter) (Watts and Strogatz, 1998). The question, however, is: what structure supports the broadest possible range of applications?

A node in an overlay network is an autonomous, networked, computational unit with a set of local resources at its disposal and attempts to fulfil a number of mission targets. To accomplish these targets, most nodes will require the resources or services of other nodes: the nodes must cooperate. This cooperation is not random: behavioural patterns can be distinguished and are captured in the concepts of group locality and time locality, which were originally formulated by Iamnitchi et al. (2002) in the context of scientific collaborations on the internet:

- Group locality - Nodes have a tendency to work in groups. Nodes with a related functionality often require clustered cooperation to fulfil their mission targets.

- Time locality - The same entity often requests the same resource within short time intervals. Though this behaviour is also reflected in the clustered cooperations of group locality, it adds another level to it. Time locality identifies those relations where a node requires the resources of a node with a different functionality.

Combining the requirements set in this section and in Section 1 with the concepts of group and time locality, semantic overlay networks are defined as follows: A semantic overlay network is a small-world overlay network which self-organises into a structure reflecting and supporting group and time locality and supports semantic routing with attribute-based addressing.

Note that group and time locality-based structures serve a dual purpose. It provides an overlay topography which increases overlay efficiency by ensuring a short path between nodes that use each other's resources while also providing a structure which can be exploited to achieve efficient semantic routing (see Section 3.5).

\section{Agora: a semantic overlay design}

This section deals with the design of a set of algorithms and techniques (Agora) that allows the construction of semantic overlay networks. 


\subsection{Semantic distance}

The key to the construction of semantic overlays is the ability to turn the semantic similarities between functionalities into numbers, i.e., a metric which yields a number proportional to the difference between the functionality of two components: semantic or functional distance.

The functionality of a node is defined by the combination of the node's resources, its mission targets and the external resources it requires to fulfil those targets. Furthermore, we require semantic overlays to support attribute-based semantic routing, which requires the description attached to messages to be compared to the descriptions of nodes. Ergo, to facilitate such a comparison, a functionality description should also be attribute-based. The most widely used attribute-based language on the internet today is the Extensible Markup Language (XML), which is a logical choice for constructing functionality descriptions.

A node functionality description is an XML file composed of two parts: the description of the node itself and a list of expressions of interest, which describe the resources and services needed during operation. The latter are XML queries which syntactically take the same form as the description of the target device itself, expanded with search masks and tags. Furthermore, all data must be marked either static or dynamic, with the latter comprising all runtime parameters (see Section 3.2 for its motivation). An example XML description file can be found in Figure 1.

The generic form of the formula to calculate the semantic distance $\delta(u, v)$ between node $u$ with description file $X M L_{u}$ and node $v$ with description file $X M L_{v}$, both conforming to the same XML schema, is then:

$$
1-\operatorname{size}\left(N_{\text {common }}\right) /\left[\operatorname{size}\left(N_{\text {common }}\right)+\operatorname{size}\left(N_{n c, x m l u}\right)+\operatorname{size}\left(N_{n c, x m l v}\right)\right] \text {, }
$$

with $N_{\text {common }}$ as the set of common XML nodes, where an XML node is defined as common if and only if the node and all its ancestors exist in both files. Text and values can only be contained by leaf nodes and count as a separate node for the purpose of the distance metric. Attributes are ignored. The symbol *, which yields an automatic match, is allowed for text fields in expressions of interest. $N_{n c, x m l}$ is the set of nonmatching nodes in XML file $i$.

This semantic distance metric is the novelty, the strength, but also the weakness of the proposed semantic overlay system. It allows the construction of a semantic overlay, but an ill-constructed XML will deteriorate that very construction. Well-designed schemas respect the rule that the depth of the data in the XML is inversely proportional to the importance of that data.

\subsection{Node links}

To enter an Agora overlay network, a node requires the address of one active node in that network. Using that entry point and the algorithms, described in Sections 3.3 and 3.4, this node will then establish four types of node links. 
Figure 1 A fragment of the XML description file of an intelligent wind turbine used within the distributed electricity generation application, realised using Agora

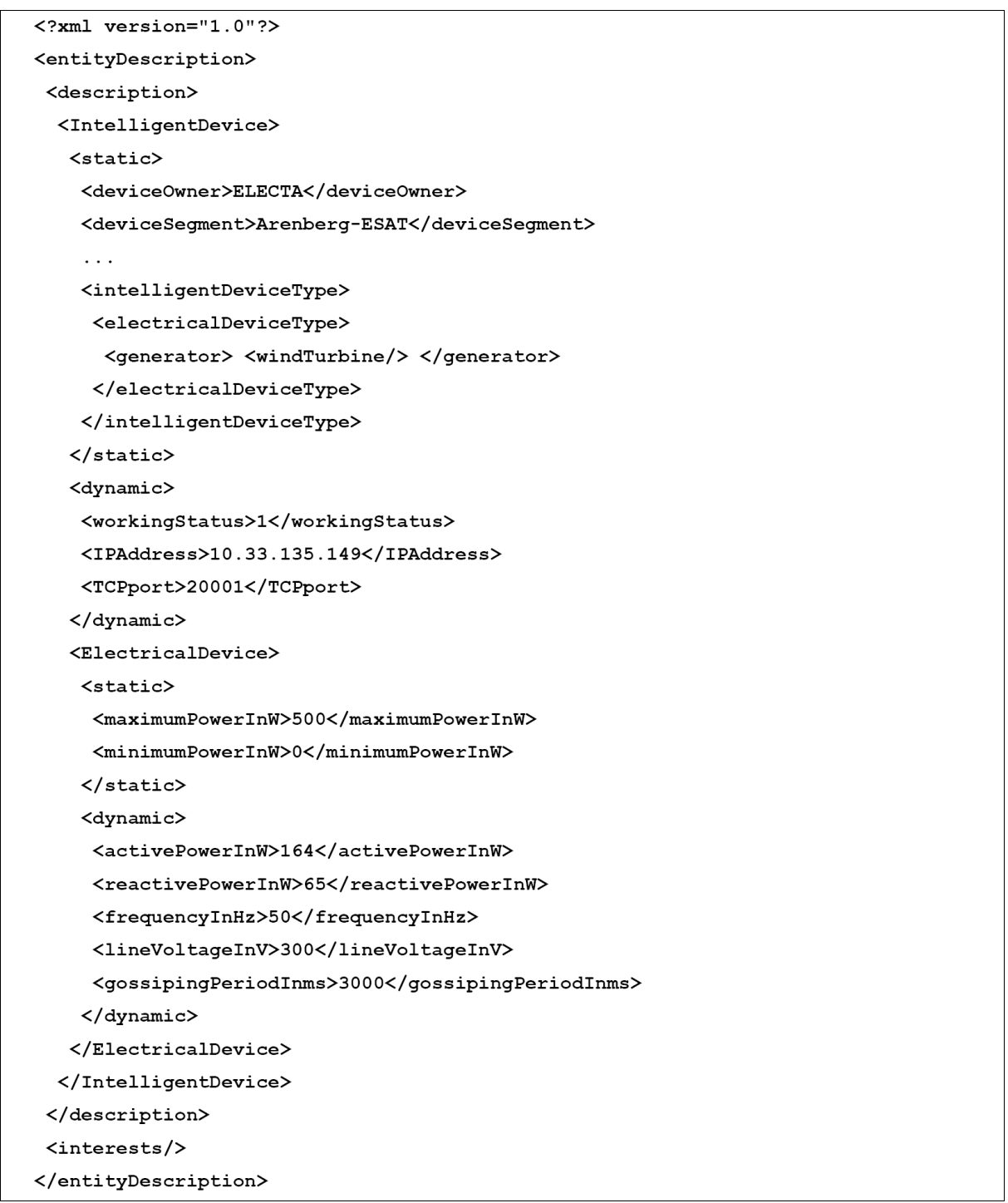

\subsubsection{Companion links}

Every Agora node forms a pre-fixed number of companion links. That means that every node $u$, which is a member of the Agora overlay network composed of the set of nodes $V$ $(|V|=n)$, has a set of companions $C_{u}$ which is of fixed size $T_{|C|}$ (provided $n$ is at least $T_{|C|}+1$ and $u$ has converged - see Section 3.3). Companions are those nodes semantically closest to $u: \forall u \in V, \forall v \in C_{u}, v \neq u, \forall w \in V \mid C_{u}, w \neq u: \delta_{c}(u, v) \leq \delta_{c}(u, w)$, with $\delta_{c}$ being the companion distance, i.e., the semantic distance calculated after all the expression of interest fields are dropped, together with the dynamic data. The latter is to ensure a stable network structure, as taking into account dynamic data, i.e., runtime 
variable data, would result in continuous overlay structure changes (the same is valid for the metrics $\delta_{p}$ and $\delta_{f l}$, which are explained below). The formation of companion links ensures that the group locality requirement is fulfilled.

Note that the companion set of a node may temporarily stray from its optimal composition due to fluctuations in the network composition (new or leaving nodes, etc.). The algorithms, described in Sections 3.3 and 3.4, provide the self-organising mechanisms to continuously repair and adapt the network to these alterations.

\subsubsection{Pupil links}

The second set of neighbours is the pupil set $P$, which is composed of the nodes semantically closest to the expressions of interest in the XML functionality description. $\forall u \in V, \forall v \in P_{u}, v \neq u, \forall w \in V \mid P_{u}, w \neq u: \delta_{p}(u, v) \leq \delta_{p}(u, w)$, with $\delta_{p}(u, v)$ as the pupil distance from node $u$ to node $v$, i.e., the smallest distance obtained by applying the semantic distance formula on the sets of XML files acquired by taking the description field of node $v$ and comparing it to the different expression of interest fields of node $u$. Additionally, as for $\delta_{c}$, all dynamic data are excluded.

The target size of $P\left(T_{|P|}\right)$ is predefined separately for each node, as it relates strongly to the application running on the node. Pupil links realise the support of time locality, as they prelink the nodes to the resources they require.

\subsubsection{Far links}

If nodes only form companion and pupil links, the resulting clustering by functionality also brings about a high probability of network partitioning. To ensure a connected graph, far links are introduced (set $F$ ). All the nodes within an Agora semantic overlay network have a probability $\mathrm{P} f l$ of constructing a far link $\left(T_{|F|} \in\{0,1\}\right)$ in addition to the other links: $\forall u \in V, \forall v \in F_{u}, v \neq u, \forall w \in V \mid F_{u}, w \neq u: \delta_{f l}(u, v) \leq \delta_{f l}(u, w)$, with $\delta_{f l}(u, v)$ as the far link distance from node $u$ to node $v$, i.e., the average distance obtained by applying the semantic distance formula on the sets of XML files acquired by taking the description field of node $v$ and comparing it to the description field and the different expression of interest fields of node $u$. As for $\delta_{c}$ and $\delta_{p}$, the dynamic data are excluded.

Far links are the equivalent of the rewired links in the small-world construction method (Watts and Strogatz, 1998), which takes a regular graph and transforms it to a small-world graph by randomly reconnecting a small percentage of links. These links provide 'shortcuts' in the network which strongly reduce the diameter, with only a small distortion of the regular structure. Consequently, the combination of companion/pupil links (functional clustering) with far links (low diameter) result in a small-world topology. Experiments show that $\mathrm{P}_{\mathrm{fl}}=0.5$ is sufficient to prevent partitioning and low enough to avoid a strong distortion of the clustered topology.

\subsubsection{Orphans and orphan links}

Orphan nodes are nodes with indegree zero, i.e., nodes which can reach other nodes, yet cannot be reached themselves. These can emerge, e.g., if a node refers to the functionally similar nodes, yet is too different from those companions for them to refer to the orphan and no far links, nor pupil links lead to it. The solution is to have each node refer to the 
$T_{|O|}$ nodes that most recently announced a link (see Section 3.4) and to which no link is active: the orphan links (set $O$, which operates as a First-In, First-Out (FIFO) buffer). Experiments show that, depending on the used XML schema, a $T_{|O|}$ equal to one or two eliminates the orphan phenomenon.

\subsection{Convergence}

After a newly entered node establishes a link to its entry point node, it starts to converge, i.e., the node progressively searches for the semantically closest nodes using $\delta_{c}, \delta_{p}$ and $\delta_{f l}$ by periodically transmitting a request message per metric. A request message is composed of the requesting node $v$ 's XML functionality description, a request threshold $\Theta$, a hoplimit $\Lambda$, the node's current associated neighbour set $C, P$ or $F$ and a list of visited nodes $V$, which is initialised with $V=\{v\}$. After transmitting the request messages, the converging node waits for a fixed period of time $\left(T_{\text {cycle }}\right)$, in which it listens for and evaluates replies with the addresses of potentially better neighbours. If that candidate neighbour is semantically closer than one of the current members of the associated set, a new link is established.

In case of a companion request $\Theta=\max \left(\forall i \in C_{v}: \delta_{c}(v, i)\right)$, unless if $|C|<T_{|C|}$, then $\Theta=\delta_{\max }$. For pupil requests, $\Theta=\max \left(\forall i \in P_{v}: \delta_{p}(v, i)\right)\left(\Theta=\delta_{\max }\right.$ if $\left.|P|<T_{|P|}\right)$ and for far link requests, $\Theta=\min \left(\forall i \in F_{v}: \delta_{f l}(v, i)\right)\left(\Theta=\delta_{f l \text { min }}\right.$ if $\left.|F|<T_{|F|}\right)$. The reason for $\Theta$ not being 1 (for companion and pupil requests) or 0 (for far link requests) if the respective set target sizes are not yet reached is to limit the initial burst of traffic that is the result of a converging node with (few) unfit neighbours making requests with high, respectively low thresholds, which results in a high number of nodes qualifying for neighbourhood membership. Instead, $\delta_{\max }$ or $\delta_{f l, \min }$ are employed, which are system parameters that must be set in the function of the used XML description set.

After the expiration of the waiting interval $T_{\text {cycle }}$, the node checks if convergence is reached, i.e., if the neighbour sets are optimal, in which case it stops. Convergence is detected if no node replied to the latest request messages. If no convergence is detected, another convergence cycle is started and new request messages are sent into the overlay network. This procedure is visualised in Figure 2, which shows a node entering an Agora overlay network and its subsequent hopping through the overlay to its optimal position. Periodically, all nodes reconverge (period $T_{\text {conv }}$ ) to allow adaptation to changing network compositions. Figures 3 and 4 contain the formalised convergence and reply processing algorithms, respectively.

Every request message is sent with a prefixed hoplimit $\Lambda$. Each time a node $u$ receives a request $\mathfrak{M}$ and its hoplimit $\Lambda_{\mathfrak{m}}\left(\Lambda_{\mathfrak{m}} \leq \Lambda\right)$ is $>0$, it will then forward the message using the request forward strategy after decrementing $\Lambda_{m}$ and completing the visited node set $(V=V \cup\{v\})$. Additionally, upon the receipt of a request, each node checks if any member of its neighbour set $N$ is semantically closer to the requester than the attached request threshold using the semantic distance metric associated with the request. If so, a reply is sent to that requester with the address of the candidate neighbour and the metric result. 
Figure 2 The graphs of a 15-node Agora network while a new node enters the overlay network

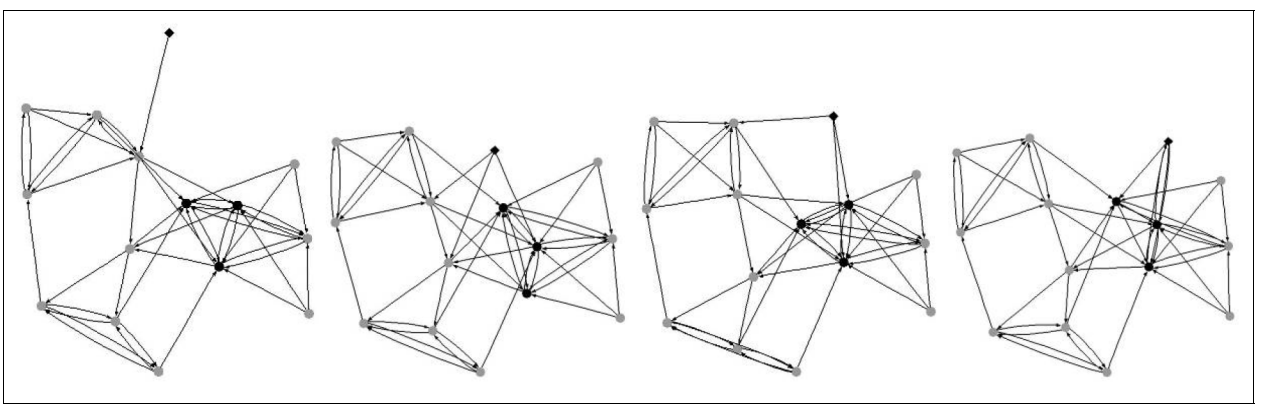

Notes: The new node (diamond-shaped) enters the overlay network (left figure), forms a link to its entry point (a random node) and starts converging. The three black nodes are the nodes semantically closest to the new node (companion nodes). The middle figures show how the node progressively 'walks' through the network towards its companions (which are reached in the right figure), after which the new node detects convergence and stops sending request messages. Note that the companions (and other nodes in the network), in turn, adapt to the newcomer and, if suited, form links to it.

Figure 3 The convergence algorithm for node $v$

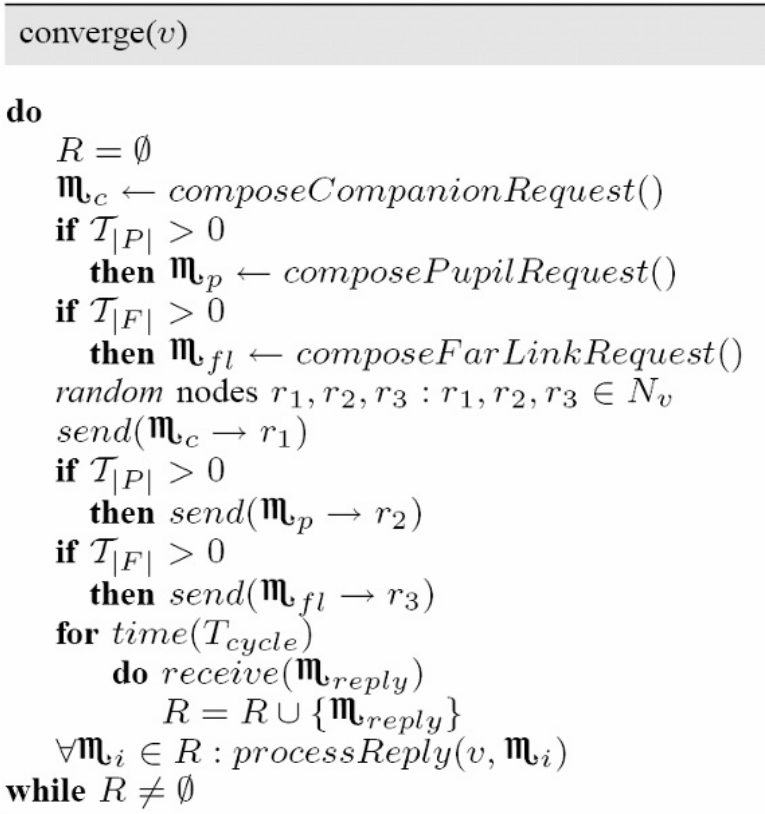

Notes: $\quad \mathfrak{M}$ symbolises a message and $N_{v}=C_{v} \cup P_{v} \cup F_{v} \cup O_{v}$ is the neighbour set of node $v$. 
Figure 4 The algorithm used to process reply message $\mathfrak{M}$ containing candidate node $w$

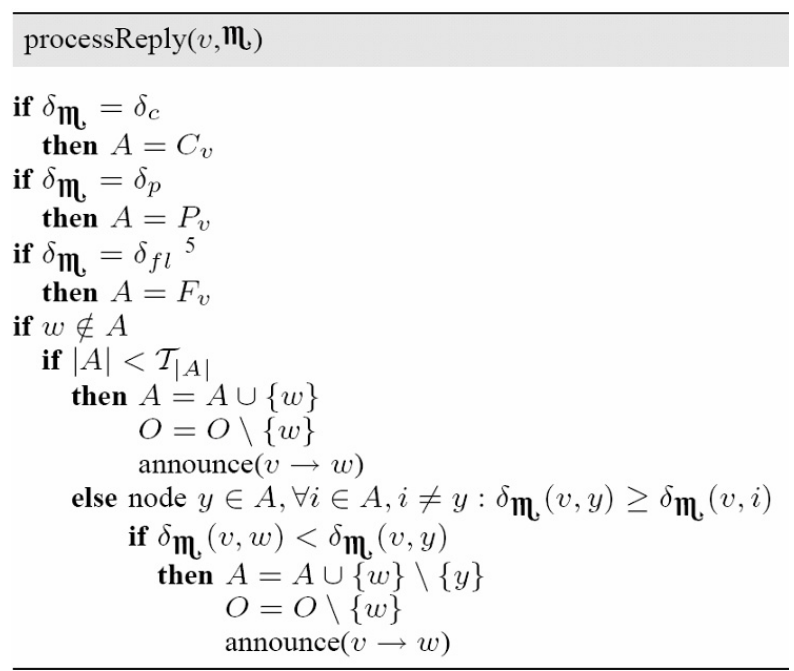

Note: $\quad \delta_{\mathfrak{M}}$ is the semantic distance metric associated with $\mathfrak{M}$.

All messages contain a unique ID, which is composed of the requester's network address, combined with a message sequence number, which is calculated using the Transmission Control Protocol (TCP) packet sequence number mechanism. All nodes have a FIFO buffer $(\mathrm{m})$ containing the last processed messages, which allows the detection and blocking of duplicate messages, e.g., to limit the overhead caused if flooding is used as a forward strategy. Figure 5 formalises the request processing algorithm.

Figure 5 The algorithm to process a request from node $u$ by node $v$

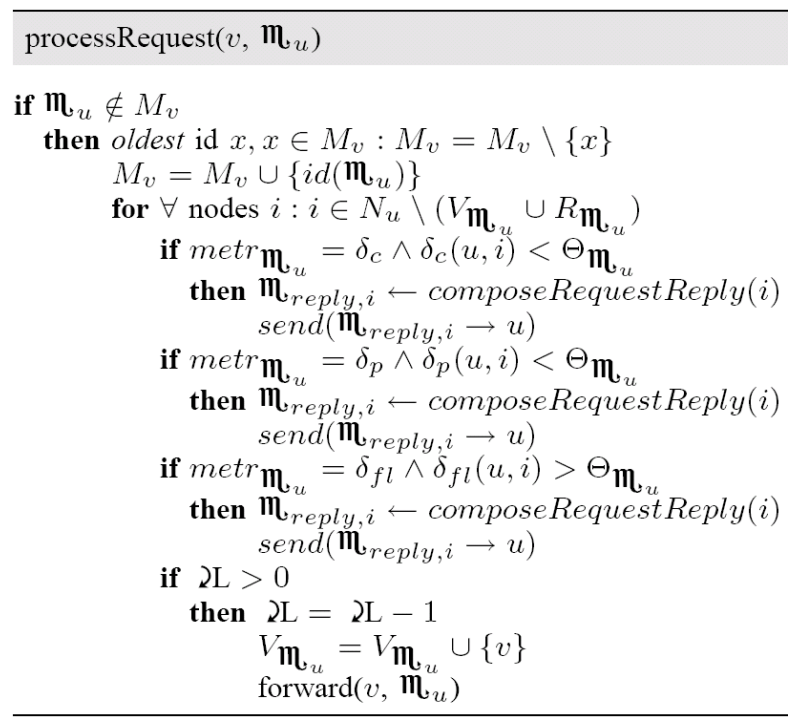


Several forward algorithms can be used, e.g., traditional blind search strategies such as flooding or random forwarding. However, it is better to use the semantic distances as a heuristic to realise a heuristic search. Indeed, the semantic distance can be used to estimate the distance to the functionality cluster the request attempts to reach. More specifically, node $u$ forwards requests to that neighbour $i$, which has not yet been visited by the request message, and which is semantically closest to requester $v$ (using the semantic distance metric associated with the request) and not yet a member of $N_{v}$. This can be written as: $i: i \in N_{u} \backslash V_{\mathfrak{M}} \backslash R_{\mathfrak{M}}, \forall j \in N u \backslash V_{\mathfrak{M}} \backslash R_{\mathfrak{M}}, j \neq i: \delta(v, \underline{i})<\delta(v, j){ }^{1}$

This results in a heuristic depth-first search with cycle-checking and without back-tracking (Poole et al., 1998).

The successive convergences of all nodes result in a self-organising system in which nodes continuously adapt to changes and, as a result of those local interactions, the network as a whole converges to a state in which nodes are clustered by functionality and linked to the clusters of their interest, while the far links ensure small-world properties. A converging self-organising system always evolves towards an attractor. However, such an attractor can be a local minimum. For a system to 'jump' out of such a local minimum, enough energy or noise must be added (Heylighen, 2003). In Agora, this energy is added by having requesters perform the first hop randomly (see Figure 3), which prevents the requests of a converged node from constantly following the same path. A second method is to increase the hoplimit, which speeds up convergence and allows nodes to sense beyond the borders of a local attractor at the cost of more network traffic. The latter is investigated in Section 4.5.

\subsection{Link announcements and dynamism}

The formation of a link consists of the node locally storing a copy of its new neighbour's description file and announcing the new link to the new neighbour. When a node receives such a link announcement, it evaluates if the originator qualifies for neighbourhood membership. The reason for this is twofold: first, it speeds up the adaptation of the overlay network to a changed situation (see, e.g., Figure 2), since otherwise, a node will only adapt to a new situation during convergence. Second, without announcements, a new node becomes an orphan permanently. A new node establishes only outgoing links and an indegree of at least one is required for requests to reach a node. If no requests reach the node, no incoming links will be established. To limit the size of an announcement message from node $u$ to node $v, \delta_{c}(v, u), \delta_{p}(v, u)$ and $\delta_{f l}(v, u)$, calculated using the locally stored XML description file of $v$ at $u$, are attached, rather than the entire description file of $u$. These metrics can also be buffered at $u$ to reduce the processing requirements.

Link announcements are periodically repeated (period $T_{a n n}$ ), as they also implement the orphan prevention mechanisms and deal with dynamism. Attached to a link announcement is the checksum of the currently stored copy of the neighbour's XML description file. Upon receiving an announcement, nodes match this checksum to their (up-to-date) XML file. If this match fails, the latest version is replied to the announcing node. Note that this is a pull system and that, hence, a delay may grow between description changes and description updates. However, the alternative to a pull system, a push system, would require all nodes to know all the nodes of which they are a neighbour. Hence, this would require a bidirectional graph, which is difficult and costly to maintain. 
The periodic announcement algorithm can be found in Figure 6; the algorithm is executed when a node receives a link announcement in Figure 7.

Figure 6 The link announcement algorithm executed by every node $u \in V$

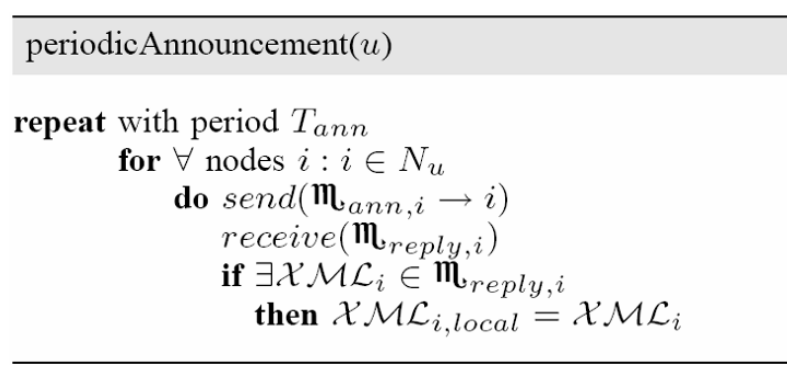

Figure 7 The algorithm a node $u$ executes upon the receipt of an announcement message from node $v$

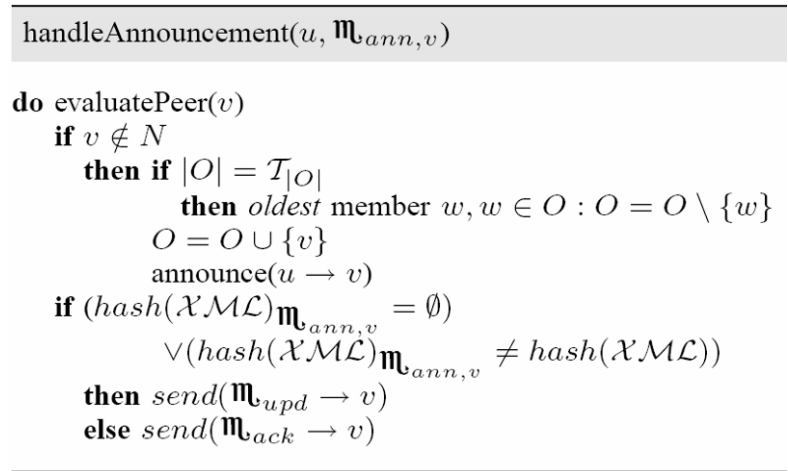

Note: The evaluatePeer(v) algorithm checks if the announcing node qualifies for companion, pupil and/or far link membership, similar to the check performed in the processReply algorithm (see Figure 4).

\subsection{Semantic routing support}

A converging node searching for semantically close nodes is a process highly similar to semantic routing. Analogous to the convergence mechanisms, the group and time locality-based structure of a semantic overlay network allows the use of semantic distance as a heuristic to realise semantic routing more efficiently than with, e.g., flooding or random walkers. Figure 8 illustrates the gain of using a heuristic search in Agora networks to realise semantic routing compared to random forwarding. Note that random walkers also gain from time locality-based structures, as the change for early hits increases when routing to announced nodes. 
Figure 8 (left) The average number of hops required for semantic routing messages to locate a matching node for Agora networks of increasing sizes, comparing random forwarding ('rand') to heuristic search (no label); (right) The average number of hops in identical circumstances, but for messages to announced nodes only
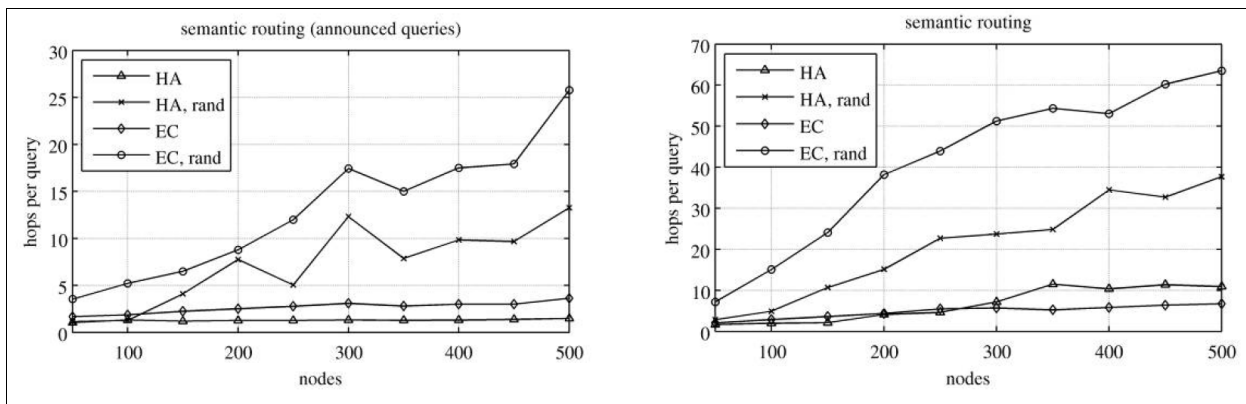

Notes: Two XML description file sets are used: HA indicates the use of a set of XML description files based on home automation devices, while EC displays the results for a set based on a combination of intelligent electricity devices and regular computational server and file-sharing resources. The data points are averages for the messages issued from and composed for each node and make up $50 \%$ of the messages to devices in which an interest was announced.

\section{Agora simulation results}

\subsection{Simulation platform}

In order to validate the claim that the presented Agora algorithms indeed result in a semantic overlay network, the simulation results are presented. Every simulation consists of the construction of a converged network, after which its properties are measured. This construction consists of the sequential addition of all nodes to the network in random order, with random time slots in between. New nodes are connected to a random active node. The simulations assume perfect components; hence, neither nodes nor communications fail. This implies a stable network composition once all nodes have been added and eventually results in a stable network structure, after which the simulation is stopped and the metrics are calculated. Stabilisation is detected if the average number of links changes (new or removed links) per time unit stays at zero for a period the length of $1.5 * T_{a n n}$.

The employed set of XML description files is composed of home automation devices. The semantic distance profile of this set is displayed in Figure 9. Every node in the set has but a small group of nodes with a small companion distance compared to the size of the set. This is intentional and the result of the XML construction rule postulated in Section 3.1. The other Agora network parameters are: $T_{|C|}=8, \delta_{\max }=0.3, \delta_{f, \min }=1.0$ and $\Lambda=4$. 
Figure 9 A histogram of all $300 * 300$ companion distances between the members of an XML description set of 300 home automation devices

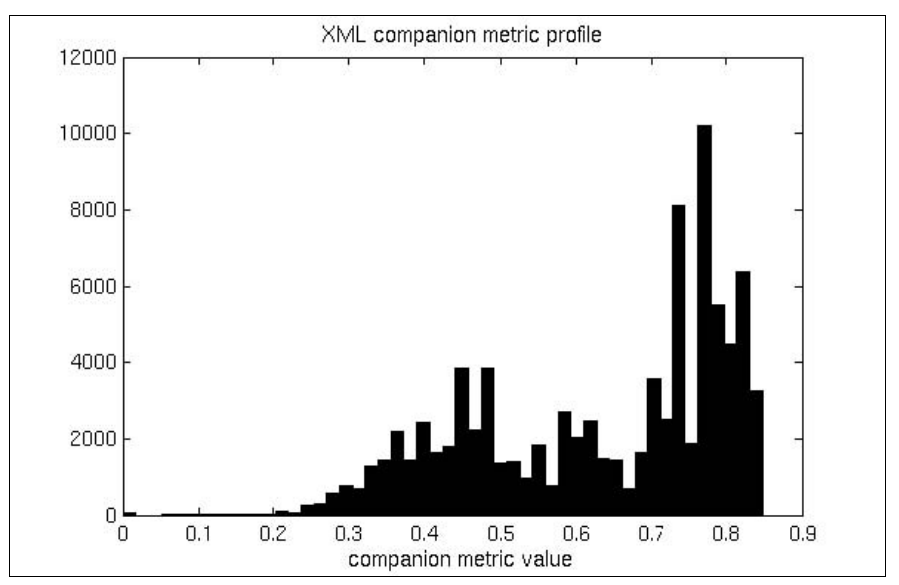

\subsection{Small-world properties}

Small-world networks are characterised by a low diameter and high clustering. An alternative metric for the diameter is the characteristic path length $\ell$, i.e., the average of the number of edges in the shortest paths between all the pairs of vertices in the graph. The structure in graphs is traditionally measured in the literature by means of the clustering coefficient $C$ : the average $C_{v}=E_{v} / K_{o, v}\left(K_{o, v}-1\right)$ for all nodes $v$ in the graph ( $K_{o}$ is the outdegree, $E_{v}$ is the number of edges that exist between these $K_{o, v}$ nodes) (Albert and Barabási, 2002). The $\ell$ and $C$ values for Agora networks with different sizes are depicted in Figure 10, which include the values for random graphs with the same average degree for comparison purposes. The $\ell$ for random graphs is proportional to the logarithm of the network size. Figure 10 confirms the same tendency of $\ell$ for Agora graphs. As for $C$, albeit its near-linear decrease with the number of nodes, the $C$ of Agora graphs is large when compared to random graphs. However, it can be argued that Agora networks of a large size will no longer maintain small-world properties due to decreasing clustering, which would limit scalability. This issue is addressed in Sections 4.3 and 4.4.

Figure 10 further displays the small-world coefficient $(S)$ for Agora networks of an increasing size and various values for $T_{|C|}$, with $S=(C / \hbar) /\left(C_{\text {rand }} / l_{\text {rand }}\right)$. Despite the decreasing $C, S$ improves linearly with the size of the network because of the faster decrease in the $C / \ell$ ratio of random networks. For comparison, the power grid of western USA has a $S$ of 10.61 and for the neural network of the nematode worm C. elegans, $S$ amounts to 4.755 (Walsh, 1999), both of which form graphs used by Watts and Strogatz as reference small-world systems, which confirms that Agora overlays do establish small-world graphs.

The classification of small-world graphs is based on the indegree distribution of those graphs. Figure 10 finally shows this distribution for a 1000-node Agora network, which matches neither the distribution of scale-free networks nor Watts and Strogatz's small-world networks (Barrat and Weigt, 2000). This confirms that semantic overlay networks, in general, and Agora networks, in particular, indeed form a new class of networks, unlike the networks previously described in the literature. 
Figure 10 (upper left) The characteristic path length /for Agora overlay networks of increasing size, together with a power curve fit $\left(y=-19.65 x^{-0.05159}+18.05\right.$, RMS error $\left.=0.041\right)$; (upper right) The clustering $C$ for Agora overlay networks of increasing size, together with a power curve fit $\left(y=-0.0001279 x^{0.9894}+0.6058\right.$, RMS error $\left.=0.0156\right)$; (lower left) The small-world coefficient $S$ for Agora networks of increasing size and various values for $T_{|C|}$; (lower right) The indegree distribution of a 1000-node Agora overlay network, including a Gaussian fit (mean $=19.85$, standard deviation $=12.5$ and RMS error $=0.0062$ )

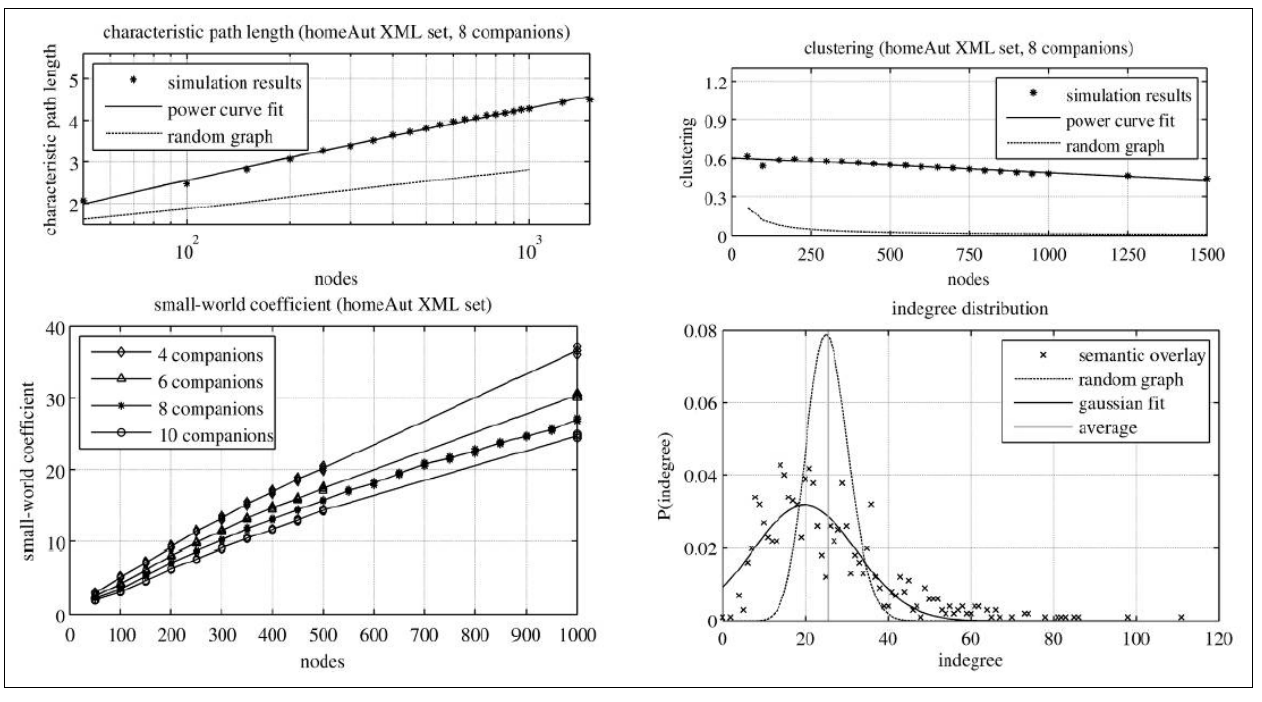

Notes: Both figures include reference values for random graphs with the same number of nodes and the same average degree as for the measurement points.

All data points in 10(a), 10(b) and 10(c) consist of the average result of ten different simulations.

The average indegree is 25.5 . The degree distribution of a random graph with the same average indegree is included for comparison.

\subsection{Group and time locality support}

Since the clustering of Agora networks decreases when the network grows, it could be claimed that its organisational properties also decrease with the number of nodes, which would imply that the structuring capacities of Agora are not scalable. However, the organisational requirements for a semantic overlay network are set by the group and time locality demands, which are badly reflected in the clustering coefficient. An overlay graph supports group and time locality if, for each node, the nodes closest in terms of companion and pupil distance are also close in terms of hop distance. The network horizon metric verifies this. Since two different variations of the generic semantic distance are used for companions and pupils $\left(\delta_{c}\right.$ and $\delta_{p}$ ), which may - depending on the used XML schema and its resulting histogram (Figure 9) - yield uncorrelated results, two different horizons are required:

1 companion horizon - The companion horizon of node $u\left(H_{c, u}\right)$ equals the shortest path which is largest in number of hops from node $u$ to the members of the node set with a $K_{o, u}$ number of nodes, which yield the smallest companion distances for node $u . K_{o, u}$ is the outdegree of $u$ 
2 pupil horizon - The pupil horizon of node $u\left(H_{p, u}\right)$ equals the shortest path which is largest in number of hops from node $u$ to the members of the node set with a $K_{o, u}$ number of nodes, which yield the smallest pupil distances for node $u . H_{p, u}$ is valid only if $T_{|P|}>0$.

The network companion horizon $\left(H_{c}\right)$ is the mean of the companion horizons of all the nodes in the network, while the network pupil horizon $\left(H_{p}\right)$ is the mean of the pupil horizons of all the nodes in the network for which $T_{|P|}>0$. The first reason to include $K_{o}$ nodes in the horizon metric is because the outdegree is independent from the network size. The second reason is because it reflects the target size of the different neighbour sets: the larger the sets and, hence, the outdegree of the nodes, the more compact a network becomes and the smaller the hop distances (at the cost of increased traffic overhead and memory usage). By using the outdegree in the horizon metrics, this effect is compensated.

$H_{c}$ and $H_{p}$ are displayed in Figure 11, which shows that the horizon for Agora networks is proportional to the logarithm of the network size. Figure 12 contains a more detailed visualisation of the evolution of the hop distance when the companion distance varies for a network of 1000 nodes, which confirms that the hop distance between nodes increases with the companion distance. The conclusion is that both the group and time locality requirements are fulfilled. Additionally, as this system-wide organisation is achieved by means of local interactions only, this also implies that the semantic overlay network requirement for self-organisation is fulfilled.

Figure 11 (left) The companion horizon $H_{c}$ for Agora networks of increasing size and various values for $T_{|C|}$; (right) The pupil horizon $H_{p}$ for Agora networks of increasing size and various values for $T_{|C|}$

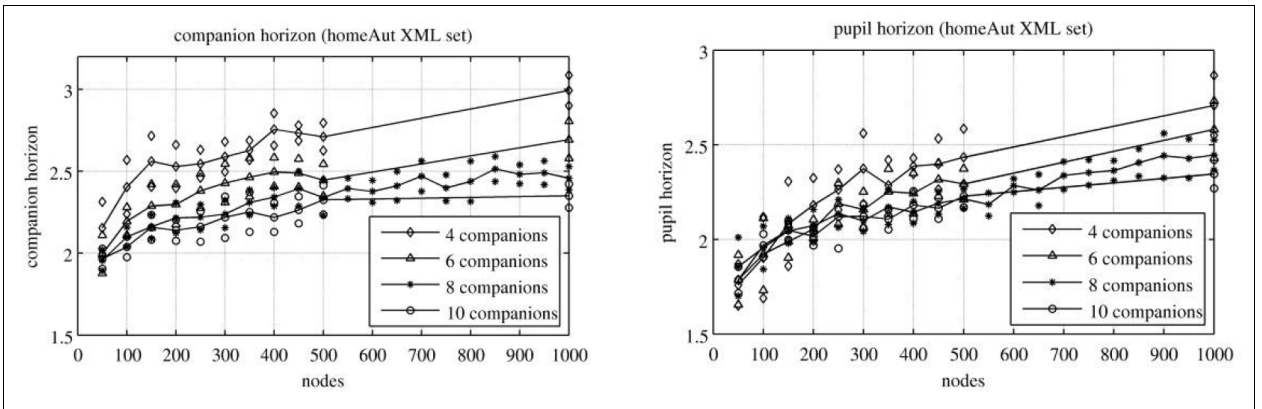

Note: Each data point consists of the average result of ten simulations and their standard deviation. 
Figure 12 (top) The sum of the number of nodes with a companion distance lower than or equal to the listed $\delta_{c}$ for all nodes to all nodes in a 1000-node Agora network; (bottom) The average hop distance to those nodes for the same network, which can be thought of as the values for a broadening companion horizon

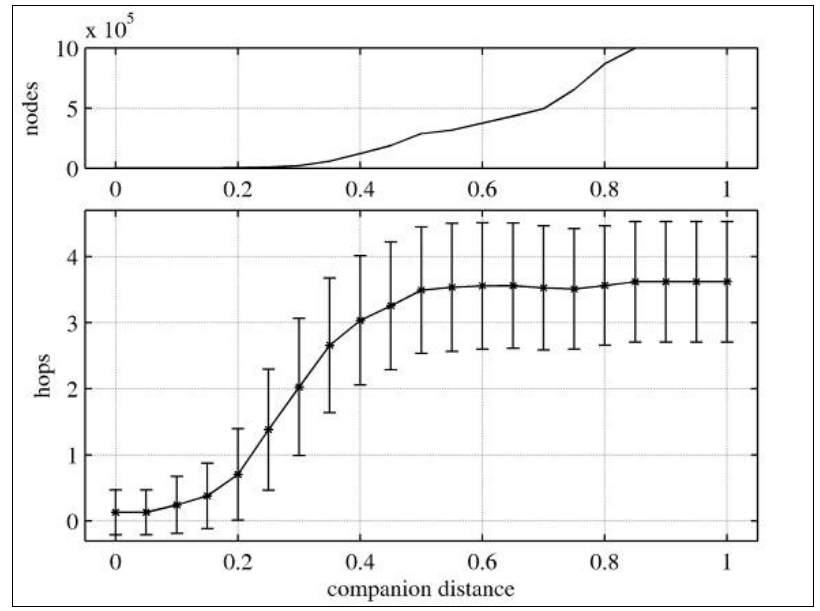

\subsection{Scalability}

A key advantage of overlay networks, when compared to centralised solutions, is their scalability. Agora's scaling properties are illustrated by the characteristic path length (Figure 10) and semantic horizons (Figure 11), which grow proportional to the logarithm of the network size, while the average outdegree (Figure 13) stays constant and the small-world coefficient (Figure 10) even increases with the number of nodes. The single dissonant is the clustering, which decreases near-linearly with the number of nodes. The clustering numerically expresses the regularity of a graph, which is a desirable property to support efficient routing within that graph. However, the structure exploited by the Agora algorithms for this purpose is the time and group locality-based organisation (see Section 3.3). As mentioned in Section 4.3, the semantic horizon metrics express this property, rather than the clustering coefficient, and these are scalable, as can be seen from the efficiency of the convergence (Section 4.5) and the semantic routing mechanisms.

Figure 13 The average outdegree $K_{o}$ for Agora networks of increasing size and various values for $T_{|C|}$

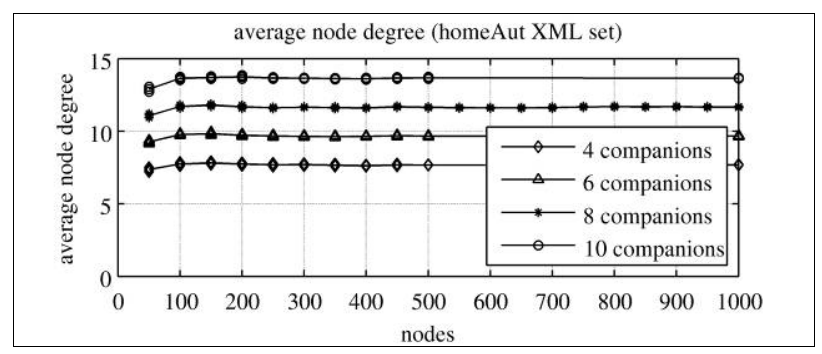

Note: Each data point consists of the average result of ten different simulations and their standard deviation. 
The most important variable by which a user can influence the properties of an Agora network is the target number of companion links, $T_{|C|}$. Incrementing $T_{|C|}$ results in the same increment on the average outdegree $K_{o}$ (Figure 13) and a nearly constant decrease of the characteristic path length of 0.1223 (standard deviation of 0.0339 ) for each unity increment of $T_{|C|}$. Also, the semantic horizons decrease as $T_{|C|}$ increases (Figure 11), while clustering increases. As such, since $/$ decreases and $C$ increases, one would expect the small-world properties of the graph to improve. This is, however, not the case, as the increase of $K_{o}$ results in a stronger increase of the $\mathrm{C} /$ /ratio for the reference random graph than for Agora graphs. Nevertheless, it can be concluded that the graph properties for Agora graphs, in general, improve if $T_{|C|}$ is increased, but not without a cost: the increase in links demands extra resources at the nodes which maintain them and results in an increase of the steady state traffic (see Section 4.6).

\subsection{Convergence performance}

The network load due to a semantic overlay network can be split into three parts: network convergence load, network maintenance load and application load. Periodic reconvergence in case of a converged stable network composition is considered network maintenance and represents, together with the link announcements, the cost of the responsiveness of the semantic overlay to changes. This network maintenance load or steady state load is investigated in Section 4.6, while this section focuses on the Agora convergence mechanisms, i.e., the costs and speed of adding a new node to an Agora overlay network when the number of nodes $\mathrm{n}$ and the hoplimit $\Lambda$ vary.

The convergence of a node is efficient if it converges fast (low number of convergence cycles before convergence) while imposing a low load on the communication network. The network load composes of all sent and forwarded request messages plus all the request reply messages that are triggered by the converging node. Convergence efficiency is numerically expressed by the convergence efficiency metric $\varepsilon: \varepsilon=1000 /(\subset * \# \overline{\mathfrak{M}})$, with (C) as the average number of cycles required for a node to converge and $\# \overline{\mathfrak{M}}$ as the average number of messages sent due to a single converging node during a single convergence cycle. Measurements must be made for newly added nodes during their initial convergence, whose entry point is randomly chosen.

A converging node jumps through the network towards an attractor. However, $\varepsilon$ does not contain any information on the quality of the attractor a node ends up in after its initial convergence. This position quality $\left(Q_{u}\right)$ of a node $u$ is defined by $N_{v}$ and the semantic distances to those neighbours and depends upon $u$ 's entry point, the network's node composition, the request forward method and $\Lambda$. This can be written as: $Q_{u}=\frac{\sum_{v \hat{v} C_{u}} \delta_{c}(u, v)+\sum_{v \hat{I} P_{u}} \delta_{p}(u, v)+\sum_{v \hat{I} F_{u}}\left(1-\delta_{f l}(u, v)\right)}{\left|C_{u}\right|+\left|P_{u}\right|+\left|F_{u}\right|}$. The average of the $Q$ of all nodes in the overlay network is then a measure of the semantic quality of the network.

$\varepsilon$ and $Q$ are depicted in Figure 14. The conclusion is that hoplimit $\Lambda$ can be used as a tradeoff parameter between the speed and network load, on the one hand, and the quality of the node's position, on the other hand. Indeed, as discussed in Section 3.3, due to the self-organising nature of Agora networks, it is possible for converging nodes to 
(temporarily) get stuck in local minima. One proposed solution was to increase the hoplimit, which increases the range of a single neighbour search. As illustrated in Figure 14, the associated cost is an increased amount of messages and a decrease of $\varepsilon$.

Figure 14 (left) The convergence efficiency $\varepsilon$ for Agora networks of increasing size and various request hoplimit values $\left(T_{|C|}=6\right.$ ); (right) The position quality $Q$ and its standard deviation for Agora networks of increasing size and various request hoplimit values $\left(T_{|C|}=6\right)$

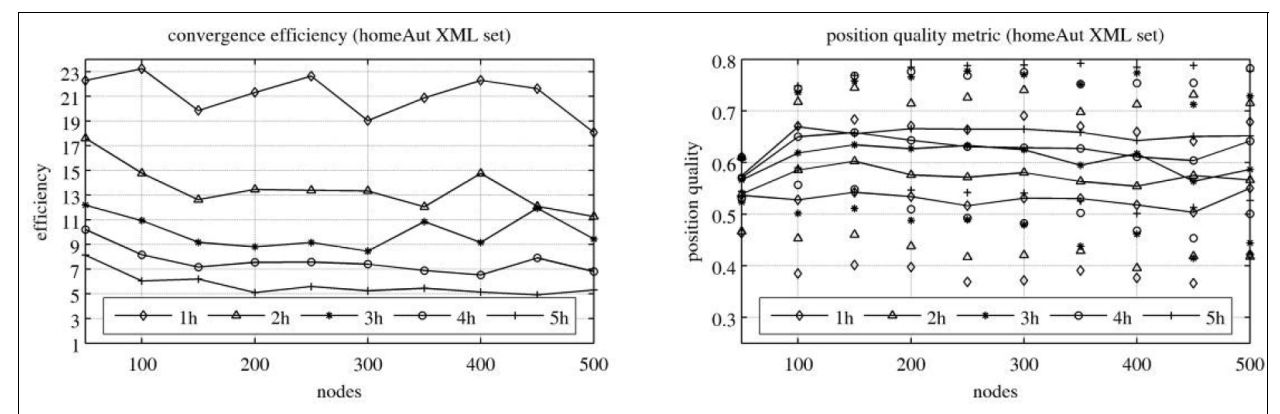

Note: Each data point consists of the average of $n^{2}$ or 10000 measurements, whichever is smaller. A single measurement consists of the $\varepsilon$ for the first convergence after the addition of a new node to a converged network, after which $Q$ is calculated for that node.

Agora networks are complex entities in which local changes may result in the structural alterations of other (distant) regions of the overlay which, in turn, exert an influence on the remainder of the overlay. The result is that node positions improve after the initial convergence, even if that convergence stopped at a local minimum. For example, for Agora networks with $\Lambda=4, Q$ increases due to the second convergence on average with 0.044 or $7.29 \%$. Hence, employing a low hoplimit does not result in a fixed inferior node position, as the node will eventually reach beyond a local minima and obtain a better set of neighbours. The cost of a low hoplimit is mainly time.

\subsection{Steady state traffic}

The composition of a semantic overlay network is defined by the nodes it contains and those node's XML description files. If this composition remains constant, i.e., no nodes join/leave the overlay, then the structure of the network as a whole converges to a stable configuration. This implies that all non-application traffic in this steady state situation, i.e., traffic caused by the announcement mechanism and the periodic reconvergences, is completely for the account of network maintenance. The steady state traffic or the average number of messages sent per node during a time $T_{a n n}$ is then: $2 K+\Lambda\left(1+P_{p}+P_{f l}\right) / c_{\text {conv }}+c_{\text {cycle }}$, with $K$ as the average node degree and $P_{p}\left(0 \leq P_{p} \leq 1\right)$ as the percentage of nodes with pupil links. $c_{\text {conv }}$ and $c_{\text {cycle }}$ are two constants such that $T_{\text {conv }}=c_{\text {conv }} . T_{\text {ann }}$ and $T_{\text {cycle }}=c_{\text {cycle }} . T_{\text {ann }}$. Expressing traffic in the function of $T_{a n n}$ allows scaling the results to any $T_{a n n}$ value (and via $c_{c o n v}$ and $c_{c y c l e}$ to any $T_{\text {conv }}$ and $T_{\text {cycle }}$ value). It also allows translation of the results of the simulations, which use units of dimensionless time, to the results in the real-time domain. 
Figure 15 depicts both the measured and estimated steady state traffic for networks of increasing size for two XML description sets: one based upon home automation devices and a second one composed of a combination of intelligent electricity devices and regular computational server and file-sharing resources. $c_{c y c l e}$ equals 0.2 and $c_{\text {conv }}$ is 4 . The average deviation between the measured and calculated results amounts to 0.029 messages per node for the home automation set and 0.024 for the electricity computation set. These small deviations are mainly due to the few request reply messages sent and due to the fact that not exactly $P_{f l} * n$ nodes will construct a far link. If the exact percentage of nodes with far links is used in the formula above, then the average deviations are reduced to 0.019 and 0.010 messages per node, respectively.

Figure 15 The average number of messages per node per time $T_{a n n}$ in a steady state regime for networks of increasing sizes, measured over a time $40 * T_{\text {ann }}$

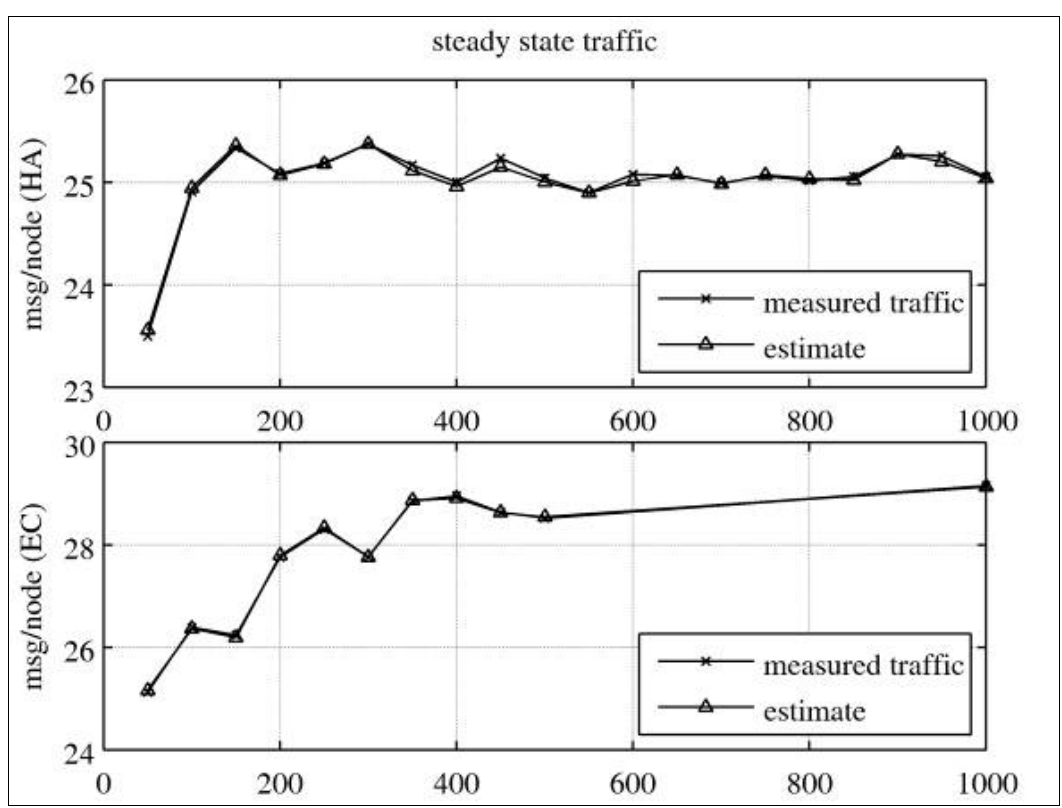

Notes: Also depicted are the estimates obtained by the steady state traffic formula.

The results at the top are for the home automation XML set (HA), while the results below are for the electricity computation XML set (EC).

$T_{|C|}=8$, hoplimit $=4$.

\section{Conclusions}

Overlay networks can support a broad distributed application range if they provide attribute-based semantic routing. However, today's designs for overlay networks focus on and are optimised for file sharing and do not support efficient attribute-based semantic routing. This paper has proposed semantic overlay networks which do allow such support, together with a design in the form of Agora algorithms. Simulations have confirmed that these algorithms result in a self-organising semantic overlay with scalable properties and the efficiency and generated traffic have been evaluated. The results confirm Agora's support of and suitability for semantic routing. 
A second primitive which should be supported by overlay networks to allow as broad an application field as possible is gossiping, a distributed data dissemination and aggregation technique (Jelasity et al., 2005). Agora's low characteristic path length suggests that it is a good infrastructure for efficient gossiping, which is confirmed by the combination being successfully used in Vanthournout et al. (2005a). However, detailed simulations are required to further investigate the suitability of Agora networks for gossiping.

Other potential optimisations include, but are not limited to:

- A node disconnection algorithm - Disconnecting nodes are now treated as failed nodes, i.e., nodes unidirectionally break links after a communication time-out.

- Kleinberg-based far links distribution - Far links from one functionality cluster have a high probability of leading to the same semantically distant cluster. Kleinberg (2004) posited that the far links or 'shortcuts' in a small-world network should be distributed uniformly over all distance scales in order to support efficient search.

- Time-locality exploitation at runtime - Voulgaris et al. (2004) proposed methods to form links to the nodes that most successfully answered queries to optimise unstructured P2P networks, i.e., exploit time locality. Agora's time locality support is based on pre-processing, yet could be completed with such methods to catch unpredicted behaviour.

Semantic overlay networks are more efficient than unstructured overlay networks, yet less than the deterministic DHT-based overlay networks. However, their added value is the broader range of supported applications, thanks to the functionality-based organisation and the resulting support of attribute-based semantic routing.

\section{Acknowledgements}

This work has partially been supported by projects EU-IST-27513 CRUTIAL and GOA/2007/09. Many thanks to the publisher for the typesetting and proofreading.

\section{References}

Aberer, K., Datta, A. and Hauswirth, M. (2004) 'Efficient, self-contained handling of identity in peer-to-peer systems', IEEE Transactions on Knowledge and Data Engineering, Vol. 16, No. 7, pp.858-869.

Albert, R. and Barabási, A-L. (2002) 'Statistical mechanics of complex networks', Reviews of Modern Physics, Vol. 74, pp.47-97.

Barrat, A. and Weigt, M. (2000) 'On the properties of small-world network models', The European Physical Journal B - Condensed Matter, Vol. 13, No. 3, pp.547-560.

Clarke, I., Sandberg, O., et al. (2001) 'Freenet: a distributed anonymous information storage and retrieval system', Lecture Notes in Computer Science, Vol. 2009, pp.46-66.

Dabek, F., Brunskill, E., et al. (2001) 'Building peer-to-peer systems with Chord, a distributed lookup service', Proc. of 8th Workshop on Hot Topics in Operating Systems (HotOSVIII), Elmau/Oberbayern, Germany, pp.81-86.

Heylighen, F. (2003) 'The science of self-organization and adaptivity', The Encyclopaedia of Life Support Systems, Eolss Publishers. 
Iamnitchi, A., Ripeanu, M. and Foster, I. (2002) 'Locating data in (small-world?) peer-to-peer scientific collaborations', 1st Int. Workshop on Peer-to-Peer Systems (IPTPS'02), Cambridge, Massachusetts.

Jelasity, M., Montresor, A. and Babaoglu, O. (2005) 'Gossip-based aggregation in large dynamic networks', ACM Trans. Comput. Syst., Vol. 23, No. 3, pp.219-252.

Kleinberg, J. (2004) 'The small-world phenomenon and decentralized search', SIAM News, Vol. 37, No. 3.

Poole, D., Mackworth, A. and Goebel, R. (1998) Computational Intelligence: A Logical Approach, Oxford University Press.

Ratnasamy, S., Francis, P., et al. (2001) 'A scalable content addressable network', Proc. of ACM SIGCOMM, San Diego, USA, pp.161-172.

Rowstron, A. and Druschel, P. (2001) 'Pastry: scalable, decentralized object location, and routing for large-scale peer-to-peer systems', IFIP/ACM Int. Conf. on Distributed Systems Platforms, Heidelberg, Germany, pp.329-350.

Vanthournout, K., De Brabandere, K., et al. (2005a) 'Agora: distributed tertiary control of distributed resources', Proc. of 15th Power Systems Computation Conf. (PSCC2005), Liège, Belgium.

Vanthournout, K., Deconinck, G. and Belmans, R. (2005b) 'A taxonomy for resource discovery', Personal and Ubiquitous Computing Journal, Vol. 9, No. 2, pp.81-89.

Voulgaris, S., Kermarrec, A-M., et al. (2004) 'Exploiting semantic proximity in peer-to-peer content searching', Proc. 10th IEEE Int. Workshop on Future Trends in Distributed Computing Systems (FTDCS), Amsterdam, The Netherlands, May.

Walsh, T. (1999) 'Search in a small world', Proc. Int. Joint Conf. on Artificial Intelligence (IJCAI), Stockholm, Sweden, August, pp.1172-1177.

Watts, D.J. and Strogatz, S.H. (1998) 'Collective dynamics of small-world networks', Nature, Vol. 393, pp.440-442.

\section{Note}

1 In case of a far link request, the expression $\delta_{f l}(v, i)>\delta_{f l}(v, j)$ is used. 